\title{
A Threshold of Meaning for Work Disability Improvement in Psoriatic Arthritis Measured by the Work Productivity and Activity Impairment Questionnaire
}

William Tillett $\cdot$ Chen-Yen Lin · Art Zbrozek • Aubrey Trevelin Sprabery • Julie Birt

Received: December 6, 2018 / Published online: June 1, 2019

(c) The Author(s) 2019

\begin{abstract}
Introduction: The Work Productivity and Activity Impairment Specific Health Problem Questionnaire (WPAI:SHP) is used to assess the impact of an intervention on work productivity in patients with psoriatic arthritis (PsA). Unfortunately, studies reporting changes or improvements in domains of WPAI:SHP by patients with PsA have a limited threshold of meaning due to the absence of published minimal clinically important differences (MCIDs). Our objective was to determine the MCIDs for improvement in WPAI:SHP in patients with active PsA.
\end{abstract}

Methods: MCIDs for WPAI:SHP domains (presenteeism, work productivity loss, and activity impairment) were derived for patients with active PsA who were biologic naïve or TNF inhibitor (TNFi) experienced using 24-week

Enhanced Digital Features To view enhanced digital features for this article go to: https://doi.org/10.6084/ m9.figshare.7982402.

W. Tillett $(\bowtie)$

Department of Rheumatology, Royal National

Hospital for Rheumatic Diseases, Bath, UK

e-mail: w.tillett@nhs.net

\section{W. Tillett}

Department of Pharmacy and Pharmacology,

University of Bath, Bath, UK

C.-Y. Lin · A. Zbrozek · A. T. Sprabery · J. Birt Eli Lilly and Company, Indianapolis, IN, USA results from two phase 3 trials (SPIRIT-P1 and SPIRIT-P2). MCIDs were derived using the anchor-based method supplemented by the distribution-based method. Anchors included achievement of the American College of Rheumatology 20 responder index (ACR20), the minimal disease activity (MDA), and the Health Assessment Questionnaire and Disability Index (HAQ-DI) MCID (improvement $\geq 0.35$ ). Anchor validity was assessed by biserial correlation and analysis of covariance modeling against the domains. MCIDs were triangulated using the receiver operating characteristic (ROC) method supplemented by the distribution-based method.

Results: The analyses included 417 biologicnaïve and 363 TNFi-experienced patients. ACR20, MDA, and HAQ-DI were valid anchors. Significant differences in WPAI:SHP domain scores were observed between patients achieving ACR20, MDA, or HAQ-DI compared to patients not achieving these clinical thresholds (all $P<0.001$ ). ROC analyses suggested that a $\geq$ $20 \%$ improvement in presenteeism, a $15 \%$ improvement in work productivity loss, and a $20 \%$ improvement in activity impairment represented clinically meaningful improvements in both populations. The distribution-based method supported the results.

Conclusion: MCIDs for the presenteeism, work productivity loss, and activity impairment domains were estimated to be $20 \%, 15 \%$, and $20 \%$, respectively, in biologic-naïve or TNFi- 
experienced PsA populations. These results will help improve the meaningfulness of WPAI:SHP improvements reported by PsA patients.

Trial Registration: SPIRIT-P1: NCT01695239, SPIRIT-P2: NCT02349295.

Funding: Eli Lilly and Company.

Keywords: Biologic naïve; Psoriatic arthritis; TNFi experienced; Work productivity; WPAI

\section{INTRODUCTION}

Psoriatic arthritis (PsA) is an inflammatory arthropathy that is typically preceded by psoriasis and affects up to $30 \%$ of patients with psoriasis [1, 2]. Physical and psychosocial aspects of the disease yield a major disease burden that impacts both quality of life and work productivity [2]. Patients with PsA have high levels of unemployment and impaired work productivity (absenteeism and presenteeism) [3]. The impact of PsA on work productivity has economic consequences, especially considering that an individual's ability to work and be productive impacts not only themselves, but also their employers and society [4]. For example, Kawalec and Malinowski reported that the cost of work disability due to PsA was \$10,754 per patient per year based upon 2013 prices [5]. Importantly, such costs, whether direct or indirect, have been demonstrated to be associated with disease activity and physical function [6].

The Work Productivity and Activity Impairment: Specific Health Problem Questionnaire (WPAI:SHP) is a version of WPAI [7] that can be modified for use with a specific disease such as PsA [8]. Patients are asked to quantify how their PsA specifically impacts their ability to work, and the questionnaire has been used in randomized controlled trials $[9,10]$ and observational studies [11]. Unfortunately, studies reporting changes or improvements in the domains of WPAI:SHP by patients with PsA have a limited threshold of meaning due to the absence of published minimal clinically important differences (MCIDs). MCIDs quantify the minimal improvement in an outcome that is perceived by patients as being beneficial, thereby translating patient-reported outcomes into clinically actionable results [12]. Thus, our objective was to determine the MCIDs for improvement in WPAI:SHP as reported by patients with active PsA who participated in two clinical trials $[9,10]$.

\section{METHODS}

\section{Study Populations and Design}

MCIDs for the presenteeism, work productivity loss, and activity impairment domains of WPAI:SHP were derived separately using the intent-to-treat populations of SPIRIT-P1 and SPIRIT-P2. These studies and their patient populations were previously described by Mease and colleagues [13] and Nash and colleagues [14]. SPIRIT-P1 and SPIRIT-P2 were multicenter, randomized, double-blind, placebo-controlled phase 3 trials. SPIRIT-P1 enrolled biologic-naïve patients. SPIRIT-P2 enrolled tumor necrosis factor inhibitor (TNFi)-experienced patients who were either inadequate responders or intolerant to TNFi therapies. Patients participating in the trials were 18 years or older, diagnosed with active PsA at least 6 months prior, met the classification criteria for psoriatic arthritis (CASPAR) criteria (at least 3/68 tender and 3/66 swollen joints), and had either a documented history of plaque psoriasis or active psoriatic skin lesions. Patients participating in SPIRIT-P1 were randomized 1:1:1:1 to receive placebo, $80 \mathrm{mg}$ ixekizumab every 4 weeks (IXEQ4W), $80 \mathrm{mg}$ ixekizumab every 2 weeks (IXEQ2W), or $40 \mathrm{mg}$ adalimumab every 2 weeks. Patients participating in SPIRIT-P2 were randomized 1:1:1 to receive placebo, IXEQ4W, or IXEQ2W. Patients receiving ixekizumab received a $160-\mathrm{mg}$ starting dose in both trials. Adalimumab was included as the activereference arm in SPIRIT-P1. Both SPIRIT-P1 and SPIRIT-P2 were conducted in accordance with the ethical principles of the Declaration of Helsinki and in compliance with local laws and regulations. All participants provided informed consent. SPIRIT-P1 and SPIRIT-P2 protocols and consent forms were approved by each site's institutional review board or ethics committee, 
including the Western Institutional Review Board for SPIRIT-P1 and the Bellberry Human Research Ethics Committee for SPIRIT-P2. The individual SPIRIT-P1 and SPIRIT-P2 sites are listed in the primary manuscript supplements $[13,14]$. Both studies were registered on ClinicalTrials.gov (SPIRIT-P1: NCT01695239, SPIRITP2: NCT02349295).

\section{WPAI:SHP}

WPAI:SHP [8] (V2.0) was administered during the double-blind treatment period (weeks 0-24) at prespecified time points, and continued in the extension periods. In the questionnaire template, "PROBLEM" was replaced with "psoriatic arthritis." The questionnaire consisted of six questions assessing employment status, hours missed from work due to PsA, hours missed from work due to other reasons, actual hours worked, the impact of PsA on work productivity, and the impact of PsA on activities outside of work. The responses to these questions were used to derive four scores: percentage of absenteeism, percentage of presenteeism, work productivity loss (incorporates absenteeism and presenteeism), and the percentage of activity impairment outside of work. Higher scores indicate a higher degree of impairment.

\section{Statistical Analyses}

MCIDs for the presenteeism, work productivity loss, and activity impairment domains of WPAI:SHP through week 24 were derived using the anchor-based method supplemented by the distribution-based method in accordance with FDA guidance [15] and Copay et al. [16]. The patient populations of SPIRIT-P1 and SPIRIT-P2 were individually pooled and kept separate for all analyses. Anchor variables included the American College of Rheumatology (ACR) 20/50/70 responder indices, the minimal disease activity (MDA) [17], and the Health Assessment Questionnaire Disability Index (HAQ-DI) MCID (improvement $\geq 0.35$ ) [13]. Efficacy results for ACR20 [13, 14], ACR50 [13, 14], ACR70 [13, 14], MDA [14, 18], and HAQ-DI MCID [13, 14] were previously
Table 1 Baseline demographics and clinical characteristics of the biologic-naïve and TNFi-experienced (inadequate responders to TNFi or TNFi-intolerant patients) patient populations

\begin{tabular}{|c|c|c|}
\hline & $\begin{array}{l}\text { Biologic } \\
\text { naïve } \\
(n=417)\end{array}$ & $\begin{array}{l}\text { TNFi } \\
\text { experienced } \\
(n=363)\end{array}$ \\
\hline Age, mean (S.D.) & $\begin{array}{l}49.5(11.9) \\
n=416\end{array}$ & $\begin{array}{l}51.9(12.0) \\
n=363\end{array}$ \\
\hline \multicolumn{3}{|l|}{ Gender, $n(\%)$} \\
\hline $\begin{array}{l}\text { Male } \\
\text { Race, } n(\%)\end{array}$ & Race, $n(\%)$ & $169(46.6)$ \\
\hline White & $\begin{array}{l}392(94.0) \\
n=417\end{array}$ & $\begin{array}{l}332(91.7) \\
n=362\end{array}$ \\
\hline $\begin{array}{l}\text { Weight }(\mathrm{kg}) \text {, mean } \\
(\mathrm{SD})\end{array}$ & $85.6(20.9)$ & $88.7(21.7)$ \\
\hline $\begin{array}{l}\text { BMI }(\mathrm{kg} / \mathrm{m} 2) \text {, mean } \\
\quad(\mathrm{SD})\end{array}$ & $\begin{array}{l}30.0(8.5) \\
n=413\end{array}$ & $\begin{array}{l}30.9(7.2) \\
n=361\end{array}$ \\
\hline $\begin{array}{l}\text { Time since PsA onset } \\
\text { (years), mean (SD) }\end{array}$ & $10.1(9.3)$ & $12.2(9.0)$ \\
\hline \multicolumn{3}{|c|}{ Prior TNFi experience, $n(\%)$} \\
\hline $\begin{array}{l}\text { Inadequate response to } \\
\qquad 1 \mathrm{TNFi}\end{array}$ & - & $204(56.2)$ \\
\hline $\begin{array}{l}\text { Inadequate response to } \\
2 \text { TNFis }\end{array}$ & - & $128(35.3)$ \\
\hline $\begin{array}{l}\text { Intolerance to a TNFi } \\
\text { cDMARD use, } n(\%)\end{array}$ & - & $31(8.5)$ \\
\hline Naïve & $61(14.6)$ & - \\
\hline Past & $89(21.3)$ & $178(49.0)$ \\
\hline Current & $267(64.0)$ & $185(51.0)$ \\
\hline $\begin{array}{l}\text { Methotrexate current } \\
\text { use, } n(\%)\end{array}$ & $226(54.2)$ & $149(41.0)$ \\
\hline $\begin{array}{l}\text { Current plaque } \\
\text { psoriasis, } n(\%)\end{array}$ & $394(94.5)$ & $339(93.4)$ \\
\hline $\mathrm{BSA} \geq 3 \%, n(\%)$ & $267(69.5)$ & $203(62.5)$ \\
\hline & $n=384$ & $n=325$ \\
\hline $\mathrm{BSA} \geq 10 \%, n(\%)$ & $\begin{array}{l}159(41.4) \\
n=384\end{array}$ & $\begin{array}{l}106(32.6) \\
n=325\end{array}$ \\
\hline
\end{tabular}


Table 1 continued

\begin{tabular}{|c|c|c|}
\hline & $\begin{array}{l}\text { Biologic } \\
\text { naïve } \\
(n=417)\end{array}$ & $\begin{array}{l}\text { TNFi } \\
\text { experienced } \\
(n=363)\end{array}$ \\
\hline $\begin{array}{c}\text { Tender joint counts (68 } \\
\text { joints), mean (S.D.) }\end{array}$ & $\begin{array}{l}20.1(13.4) \\
n=417\end{array}$ & $\begin{array}{l}23.4(15.9) \\
n=362\end{array}$ \\
\hline $\begin{array}{l}\text { Swollen joint counts } \\
\text { (66 joints), mean } \\
\text { (S.D.) }\end{array}$ & $\begin{array}{l}11.0(7.4) \\
n=417\end{array}$ & $\begin{array}{l}12.3(10.3) \\
n=362\end{array}$ \\
\hline HAQ-DI, mean (S.D.) & $\begin{array}{l}1.17(0.6) \\
n=405\end{array}$ & $\begin{array}{l}1.20(0.6) \\
n=357\end{array}$ \\
\hline SF-36, mean (S.D.) & & \\
\hline PCS & $\begin{array}{l}33.6(9.0) \\
n=404\end{array}$ & $\begin{array}{l}34.3(8.9) \\
n=354\end{array}$ \\
\hline MCS & $\begin{array}{l}47.1(11.9) \\
n=404\end{array}$ & $\begin{array}{l}48.9(12.0) \\
n=354\end{array}$ \\
\hline Employment status, $n$ (\% & & \\
\hline $\begin{array}{l}\text { Work for pay (full } \\
\text { time) }\end{array}$ & $211(50.6)$ & $162(44.6)$ \\
\hline $\begin{array}{l}\text { Work for pay (part } \\
\text { time) }\end{array}$ & $47(11.3)$ & $37(10.2)$ \\
\hline $\begin{array}{l}\text { Unable to work (due to } \\
\text { PsA) }\end{array}$ & $41(9.8)$ & $50(13.8)$ \\
\hline $\begin{array}{l}\text { Unable to work (other } \\
\text { than PsA) }\end{array}$ & $25(6.0)$ & $24(6.6)$ \\
\hline Other & $93(22.3)^{\mathrm{a}}$ & $90(24.8)^{b}$ \\
\hline WPAI, mean (S.D.) & & \\
\hline Absenteeism & $\begin{array}{l}8.6(22.3) \\
n=228\end{array}$ & $\begin{array}{l}10.8(26.0) \\
n=188\end{array}$ \\
\hline Presenteeism & $\begin{array}{l}36.6(23.8) \\
n=220\end{array}$ & $\begin{array}{l}40.8(26.6) \\
n=176\end{array}$ \\
\hline Work productivity loss & $\begin{array}{l}38.9(24.9) \\
n=220\end{array}$ & $\begin{array}{l}42.5(27.7) \\
n=176\end{array}$ \\
\hline
\end{tabular}

Table 1 continued

\begin{tabular}{lll}
\hline & $\begin{array}{l}\text { Biologic } \\
\text { naïve } \\
(n=417)\end{array}$ & $\begin{array}{l}\text { TNFi } \\
\text { experienced } \\
(n=363)\end{array}$ \\
\hline Activity impairment & $\begin{array}{l}47.0(25.1) \\
n=405\end{array}$ & $\begin{array}{l}n 2.4(25.8) \\
n=353\end{array}$ \\
\hline
\end{tabular}

HAQ-DI Health Assessment Questionnaire and Disability Index, MCS mental component score, PCS physical component score, SF-36 Short Form 36 Health Survey, TNFi tumor necrosis factor inhibitor, WPAI Work Productivity and Activity Impairment Questionnaire

a Includes volunteer, keeping house, retired, or student

b Includes keeping house, retired, or missing

published. Per FDA guidance [15], a valid anchor is expected to be understandable, interpretable, and adequately associated with the WPAI:SHP instrument. ACR20/50/70, MDA, and HAQ-DI were considered to be understood and interpretable by rheumatologists for the purposes of these analyses. To validate our anchor choices in preparation for anchor-based analyses, we evaluated associations between WPAI:SHP domain scores and anchors using biserial correlation, logistic modeling, and analysis of covariance (ANCOVA). A threshold of 0.371 signified a large effect for the biserial correlation analysis [19]. The concordance index (ranging from 0.5 to 1.0 ) from a logistic model is another metric that was used to quantify the association between anchor and WPAI:SHP domains. The larger the concordance index, the stronger the association. The ANCOVA models were employed to demonstrate a significantly greater improvement in the WPAI:SHP domains among patients who met the anchor than among those who did not meet the anchor. For the anchor-based method, we utilized the receiver operating characteristic (ROC) method to identify cutoffs for each WPAI:SHP domain that best differentiated each anchor. When a cutoff is set too low, we would expect to observe a high sensitivity and a negative predictive value. On the other hand, when a cutoff is set too high, both the sensitivity and the negative prediction value decrease while the specificity and positive predictive value increase. An ideal cutoff should balance the 
- Biologic-Naive - TNFi-Experienced

A

\section{Presenteeism}

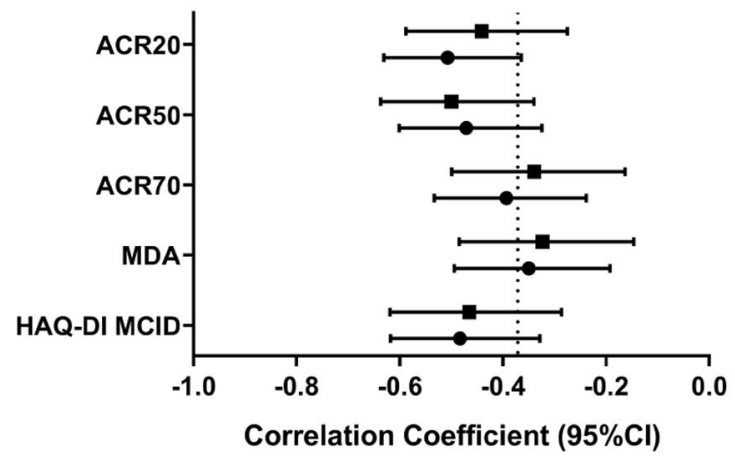

B

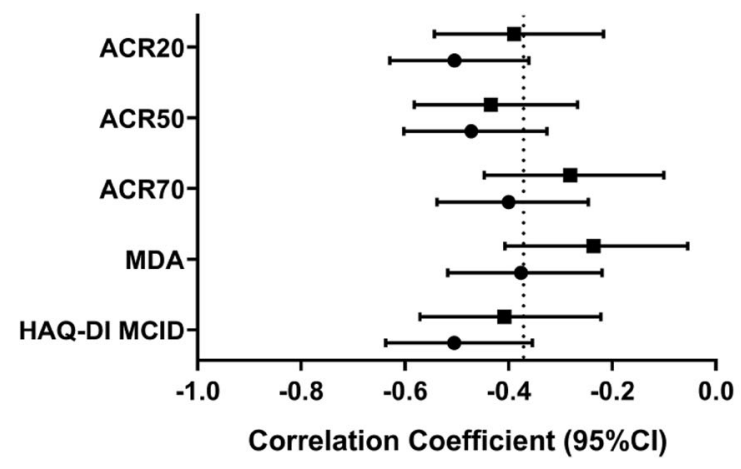

C

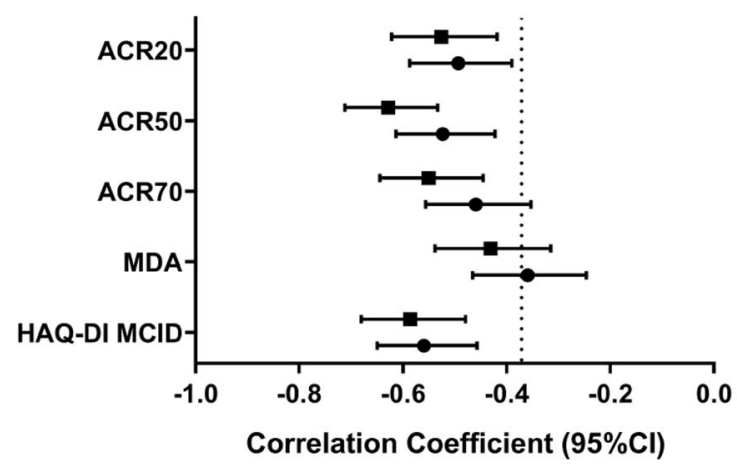

tradeoff while being greater than the half the standard deviation or SEM recommended by the distribution-based method. The distribution method used measures of instrument variability, including the standard error of measurement with an assessment of the reliability coefficient with a lower bound of 0.7 , as
4 Fig. 1 Anchor evaluation by biserial correlation analyses of ACR20, ACR50, ACR70, MDA, and HAQ-DI MCID (improvement $\geq 0.35$ ) for the presenteeism (a), work productivity loss $(\mathbf{b})$, and activity impairment (c) domains of WPAI:SHP in patients with PsA who were biologic naïve or TNFi experienced (inadequate responders to TNFi or TNFi-intolerant patients). The dotted line corresponds to a correlation coefficient threshold of 0.371. HAQ-DI Health Assessment Questionnaire and Disability Index, $M C I D$ minimal clinically important difference, $M D A$ minimal disease activity, TNFi tumor necrosis factor inhibitor, WPAI:SHP Work Productivity and Activity Impairment:Specific Health Problem Questionnaire

reported by Crawford and colleagues [20], and multiplying by half of the standard deviation [21]. The results of the anchor- and distributionbased method were used to triangulate MCIDs for the domains of WPAI:SHP [22]. Analyses were performed using SAS (version 9.4).

\section{RESULTS}

WPAI:SHP estimates were derived using previously published results $[9,10]$. The intent-totreat populations of SPIRIT-P1 and SPIRIT-P2 included 417 biologic-naïve and 363 TNFi-experienced patients with PsA, respectively. Baseline demographics and clinical characteristics were previously published for these patients $[13,14]$, and are provided in Table 1.

It was hypothesized that ACR20, ACR50, ACR70, MDA, and HAQ-DI MCID were valid anchors to use to estimate the MCIDs of WPAI:SHP using the anchor-based method. These were selected as candidate anchors because they are well understood and easy to interpret. To determine anchor validity, we tested the anchors by correlation (Fig. 1a-c) and logistic regression (Fig. $2 \mathrm{a}-\mathrm{c}$ ) modeling against the presenteeism, work productivity loss, and activity impairment domains of WPAI:SHP in the biologic-naïve and TNFi-experienced populations. The hypothesized anchors were found to be valid by both correlation and logistic regression analyses for both patient populations. 

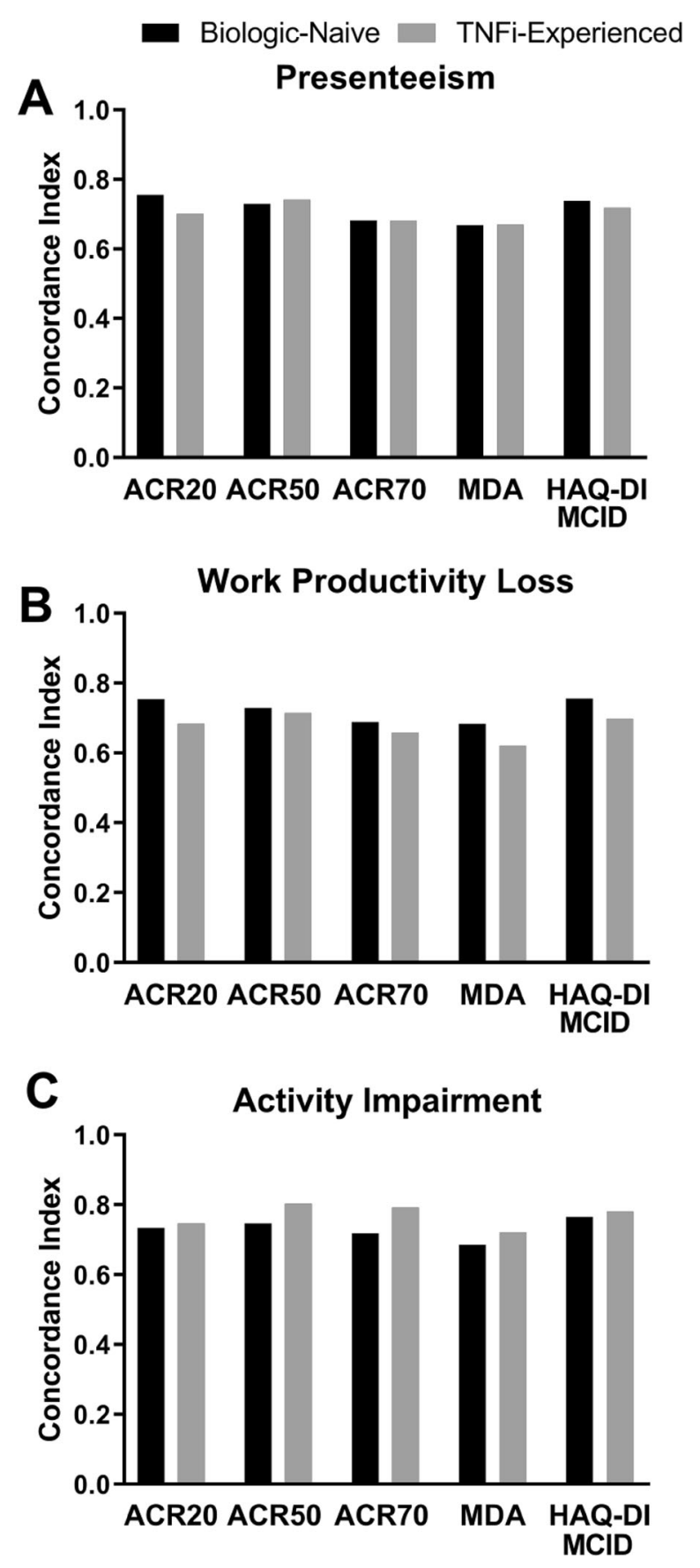

Anchors were then investigated further using an ANCOVA model to determine if there were statistically significant differences between patients who met anchor clinical thresholds and those who did not for the presenteeism, work productivity loss, and activity impairment domains of WPAI:SHP (Fig. 3a-c). Statistically significant differences were observed for all
4 Fig. 2 Anchor evaluation by logistic modeling of ACR20, ACR50, ACR70, MDA, and HAQ-DI MCID for the presenteeism (a), work productivity loss $(\mathbf{b})$, and activity impairment (c) domains of WPAI:SHP in patients with PsA who were biologic naive or TNFi experienced (inadequate responders to TNFi or TNFi-intolerant patients). HAQ-DI Health Assessment Questionnaire and Disability Index, $M D A$ minimal disease activity, $M C I D$ minimal clinically important difference, $T N F i$ tumor necrosis factor inhibitor, WPAI:SHP Work Productivity and Activity Impairment: Specific Health Problem Questionnaire

investigated anchors across the WPAI:SHP domains in the biologic-naïve and TNFi-experienced populations.

Using multiple validated anchors, ROC analyses were performed to identify a meaningful improvement in a WPAI:SHP domain that was best associated with the anchor status (Figs. 4, 5, 6). When ACR20, MDA, and HAQ-DI MCID were incorporated into ROC analyses, the suggested MCID for presenteeism was $20 \%$, that for work productivity loss was $15 \%$, and that for activity impairment was $20 \%$ for any of the tested anchors in the biologic-naïve and TNFiexperienced populations. The observed consistency across anchors highlights the robustness of these MCID estimates. In addition, these results are supported by the distribution-based method, since both half standard deviation and standard error of measurement estimates for the work productivity loss and activity impairment domains in the two populations indicate that improvements of greater than approximately $12-15 \%$ would be considered statistically significant (Table 2).

\section{DISCUSSION}

Using data from randomized controlled trials, MCIDs for the presenteeism, work productivity loss, and activity impairment domains of WPAI:SHP in patients with PsA were estimated using the anchor-based method with support from the distribution-based method. A 15\% improvement was estimated to be the MCID for 


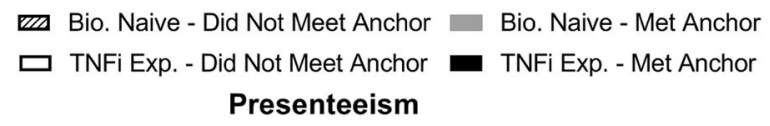

A ACR20 ACR50 ACR70 MDA HAQ-DI

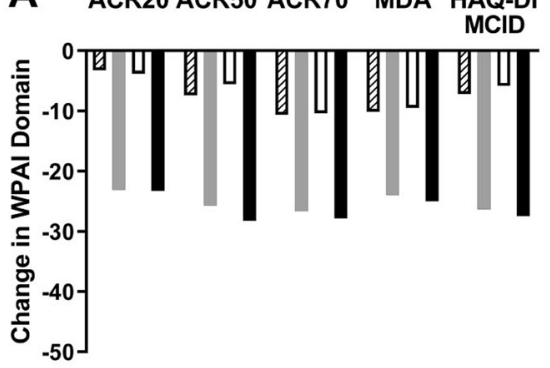

Work Productivity Loss

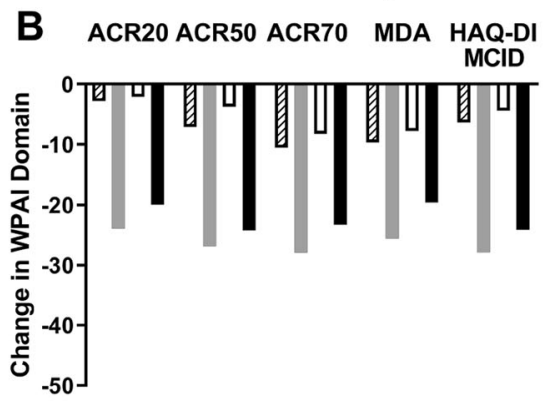

Activity Impairment
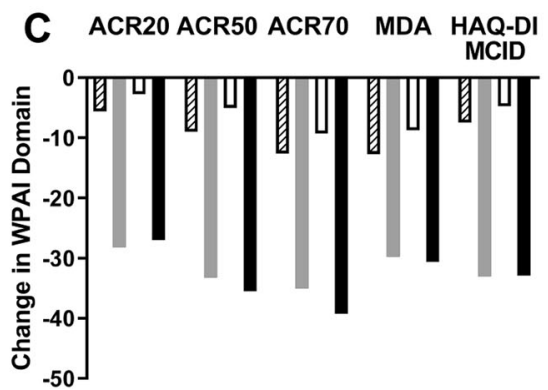

Fig. 3 Anchor evaluation by analysis of covariance (ANCOVA) modeling for the presenteeism (a), work productivity loss $(\mathbf{b})$, and activity impairment (c) domains of WPAI:SHP in biologic-naïve and TNFi-experienced patients (inadequate responders to TNFi or TNFi-intolerant patients). Domain change from baseline at week 24 was stratified by anchor achievement status, adjusting for baseline WPAI:SHP domain score. All comparisons between those who met and those who did not meet the anchors within a population group had $P<0.001$. HAQ$D I$ Health Assessment Questionnaire and Disability Index, $M C I D$ minimal clinically important difference, $M D A$ minimal disease activity, TNFi tumor necrosis factor inhibitor, WPAI:SHP Work Productivity and Activity Impairment: Specific Health Problem Questionnaire work productivity loss in either the biologicnaïve or TNFi-experienced population. A 15\% improvement in work productivity loss translates into a gain of $\$ 7593$ per patient per year in indirect costs, assuming a patient earns the 2017 mean US annual salary $(\$ 50,620)$ and that the treatment effect is maintained for 1 year $[23,24]$. A $20 \%$ improvement was estimated to be the MCID for presenteeism and activity impairment in either the biologic-naïve or TNFi-experienced population. This estimate for activity impairment translates into a 20\% improvement in non-work-related activity productivity. Importantly, we highlight that these estimates were consistent in the two patient populations.

The availability of these estimates will provide clinicians and payers investigating the impact of treatments on presenteeism, work productivity loss, and activity impairment with a greater threshold of meaning. This is important given that trials of novel therapies for PsA have used WPAI:SHP to measure the effect of treatment on presenteeism, work productivity, and activity impairment, and have reported changes for presenteeism, work productivity loss, and activity impairment but, to date, have not had a PsA-specific MCID to benchmark $[9,10]$. The use of MCIDs is paramount since MCIDs provide clinicians and payers with thresholds that identify minimum changes due to a treatment or intervention that are viewed by patients as being beneficial [16]. In addition, these MCIDs will address recent FDA guidance recommending the use of MCIDs or "responder definitions" for the interpretation of patientreported outcome results from clinical trials [15].

Recently Wu and colleagues reported MCIDs for the work productivity loss and activity impairment domains of WPAI for psoriasis (WPAI:PsO) in patients with psoriasis [25]. The MCID estimates for work productivity loss and activity impairment in patients with psoriasis were both 20\% [25]. The MCID estimate for activity impairment was identical to our estimate for patients with PsA, while the estimate for work productivity loss was $5 \%$ less. The differences observed in the estimates for work productivity loss were not unexpected given 


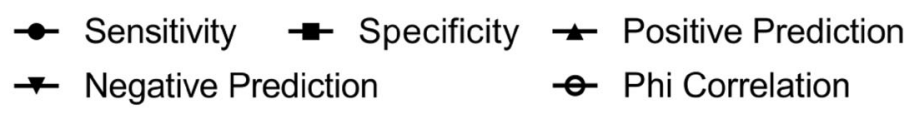

\section{Biologic-Naive}

A

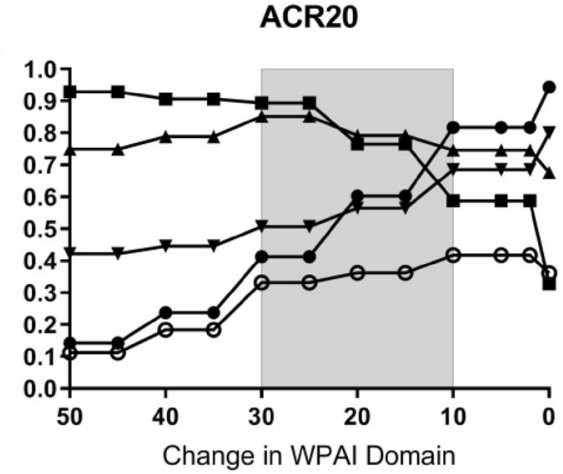

B

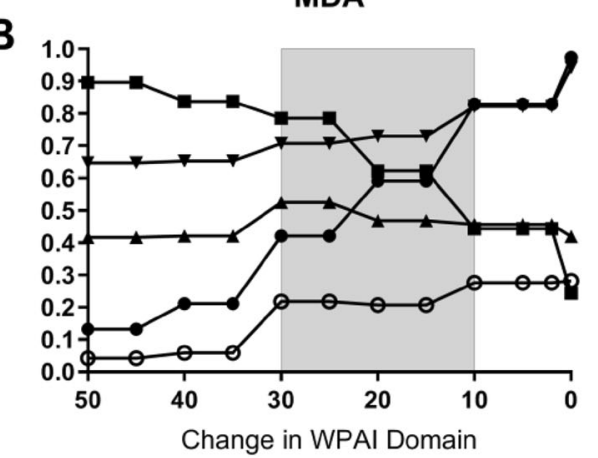

C

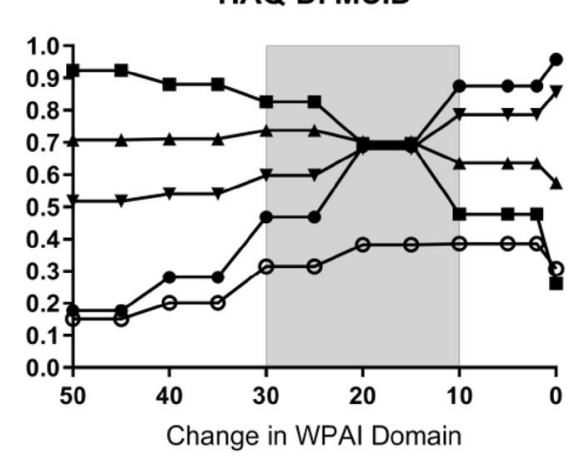

Fig. 4 Responder definitions of the WPAI:SHP presenteeism domain as determined by the receiver operating characteristic (ROC) method using WPAI:SHP domain changes from baseline at week 24 for ACR20 (a), MDA (b), and HAQ-DI MCID (c) in biologic-naïve patients and ACR20 (d), MDA (e), and HAQ-DI MCID (f) in TNFi-experienced patients (inadequate responders to TNFi or TNFi-intolerant patients). MCIDs were derived
D

\section{TNFi-Experienced}

ACR20

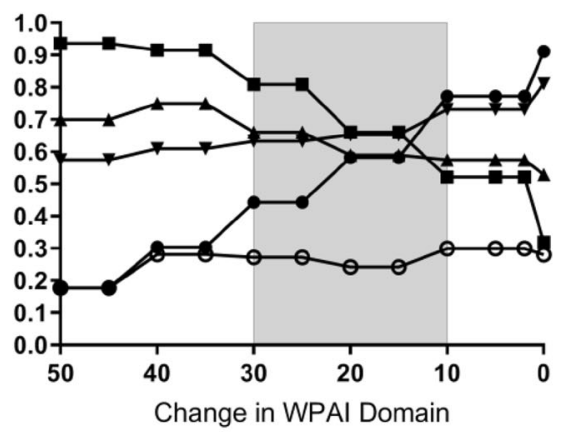

E

MDA

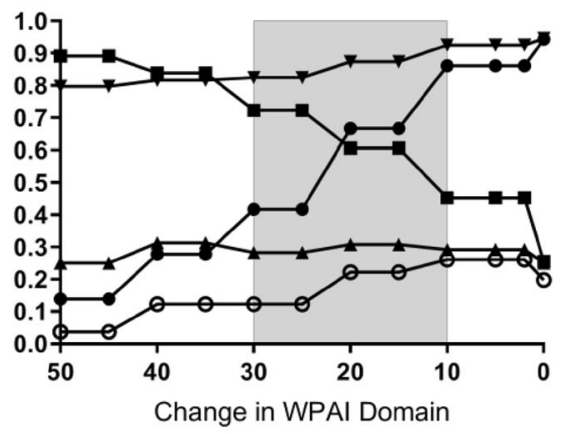

F

HAQ-DI MCID

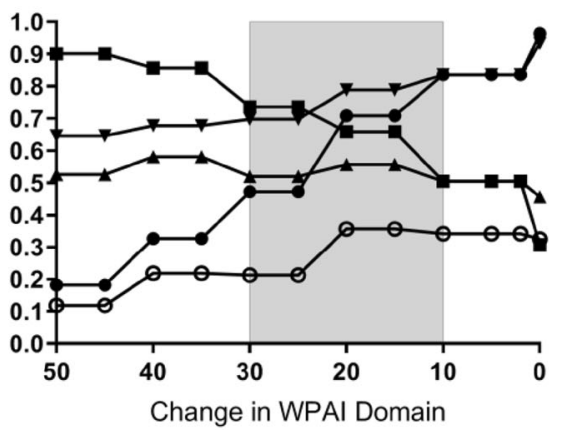

from the region reflecting a balance between specificity and sensitivity, with the lower limit defined by half the standard deviation of the distribution-based method. $H A Q-D I$ Health Assessment Questionnaire and Disability Index, $M C I D$ minimal clinically important difference, $M D A$ minimal disease activity, TNFi tumor necrosis factor inhibitor, WPAI:SHP Work Productivity and Activity Impairment: Specific Health Problem Questionnaire 


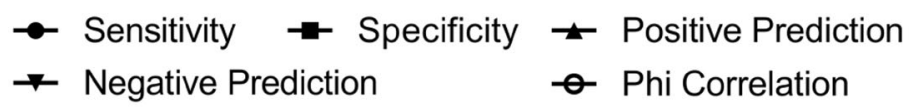

\section{Biologic-Naive}

A
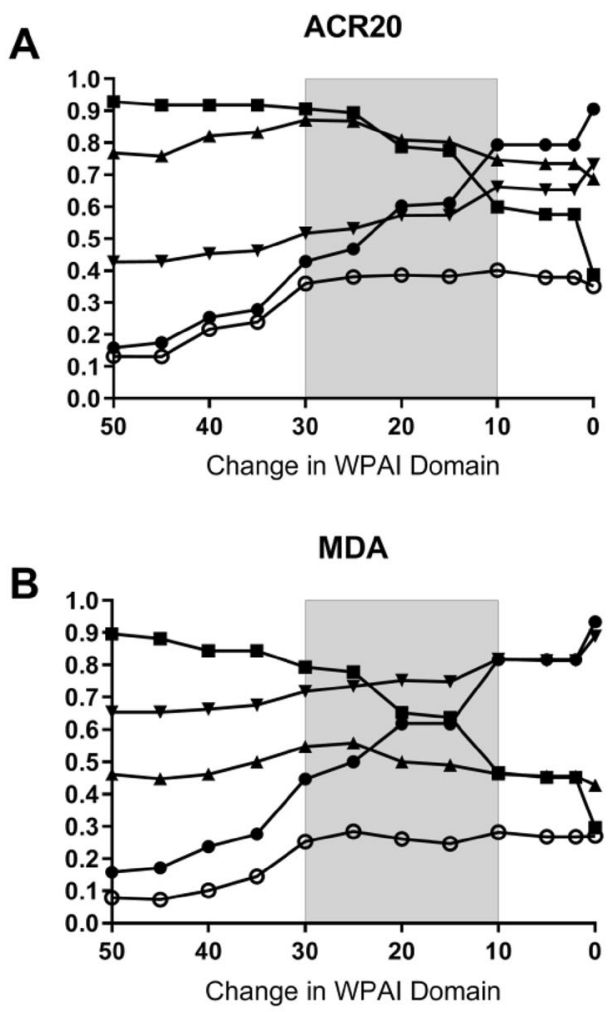

C

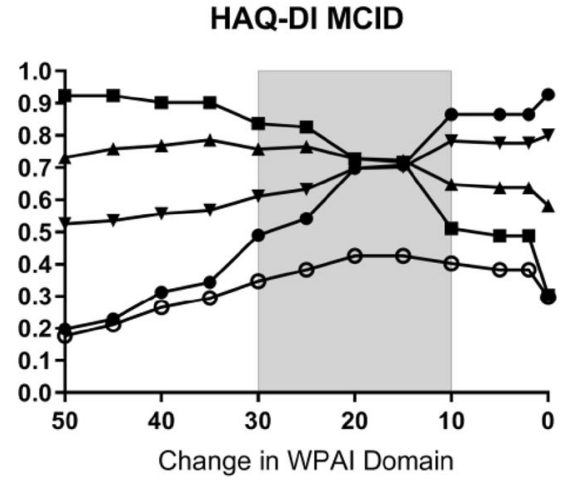

Fig. 5 Responder definitions of the WPAI:SHP work productivity loss domain as determined by the receiver operating characteristic (ROC) method using WPAI:SHP domain changes from baseline at week 24 for ACR20 (a), MDA (b), and HAQ-DI MCID (c) in biologic-naïve patients and ACR20 (d), MDA (e), and HAQ-DI MCID (f) in TNFi-experienced patients (inadequate responders to TNFi or TNFi-intolerant patients). MCIDs were derived from the region reflecting a balance between
TNFi-Experienced

D ACR20

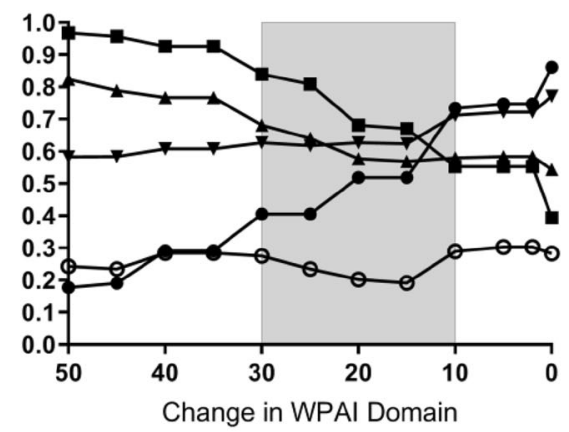

E

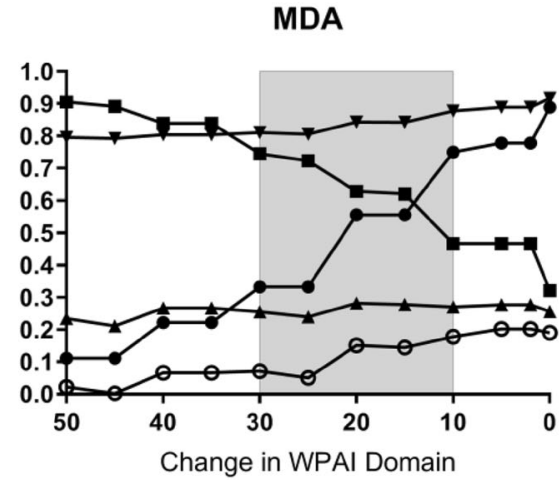

F

HAQ-DI MCID

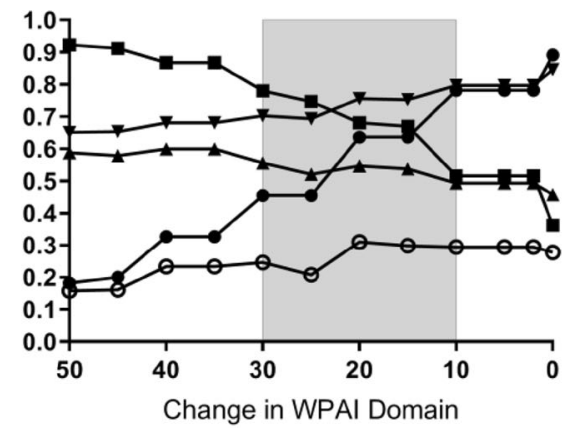

specificity and sensitivity, with the lower limit defined by half the standard deviation of the distribution-based method. HAQ-DI Health Assessment Questionnaire and Disability Index, $M C I D$ minimal clinically important difference, $M D A$ minimal disease activity, $T N F i$ tumor necrosis factor inhibitor, WPAI:SHP Work Productivity and Activity Impairment: Specific Health Problem Questionnaire 


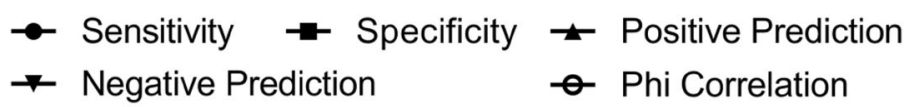

Biologic-Naive
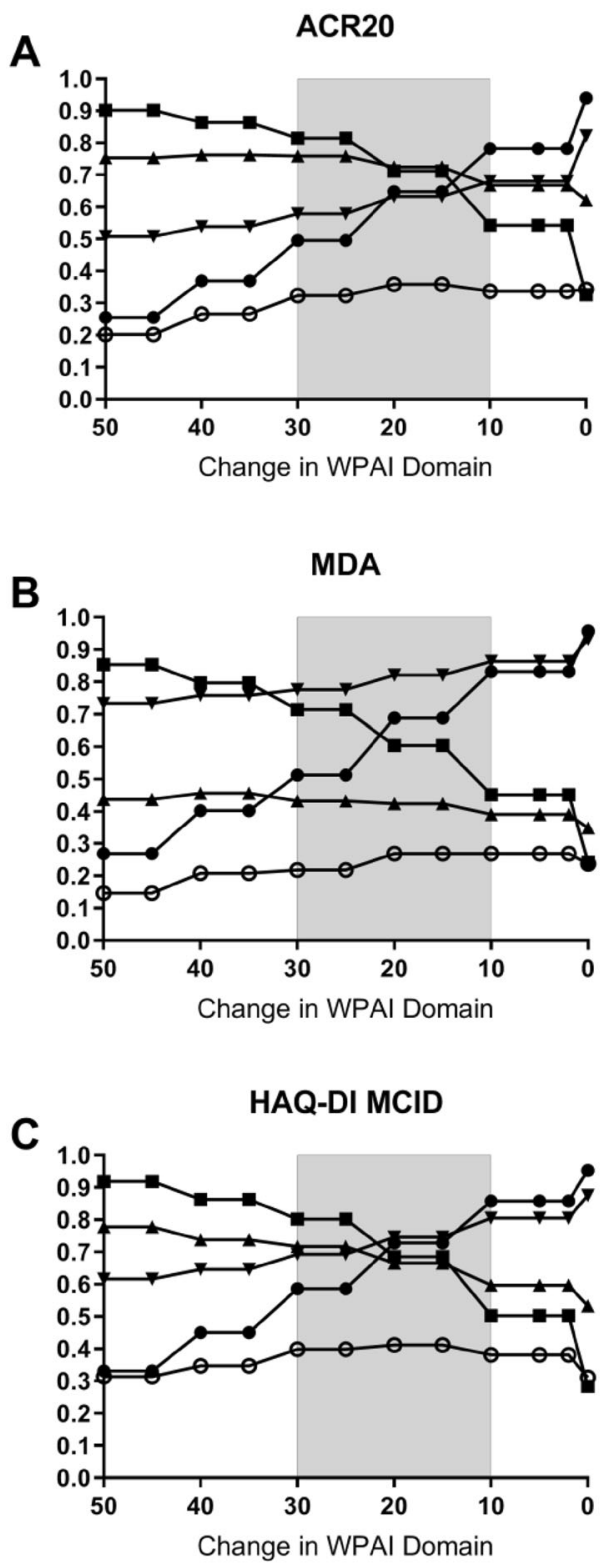

Fig. 6 Responder definitions of the WPAI:SHP activity impairment domain as determined by the receiver operating characteristic (ROC) method using WPAI:SHP domain changes from baseline at week 24 for ACR20 (a), MDA (b), and HAQ-DI MCID (c) in biologic-naive patients and ACR20 (d), MDA (e), and HAQ-DI MCID $(\mathbf{f})$ in TNFi-experienced patients (inadequate responders to TNFi or TNFi-intolerant patients). MCIDs were derived from the region reflecting a balance between

\section{TNFi-Experienced}

D ACR20

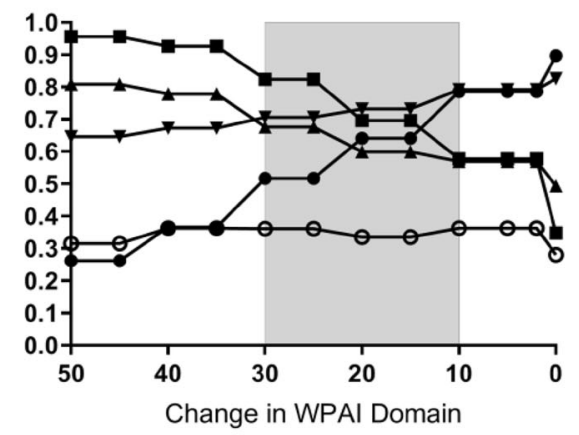

E

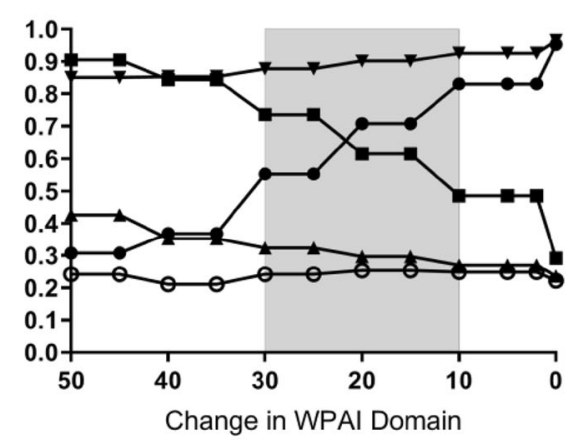

HAQ-DI MCID

$\mathbf{F}$

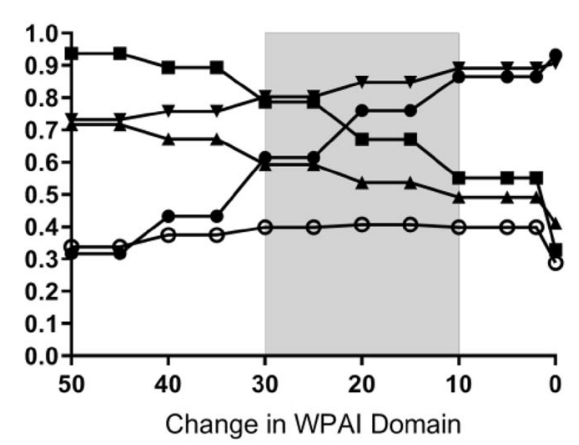

specificity and sensitivity, with the lower limit defined by half the standard deviation of the distribution-based method. HAQ-DI Health Assessment Questionnaire and Disability Index, $M C I D$ minimal clinically important difference, $M D A$ minimal disease activity, $T N F i$ tumor necrosis factor inhibitor, WPAI:SHP Work Productivity and Activity Impairment: Specific Health Problem Questionnaire 
Table 2 Distribution-based method results for the work productivity loss and activity impairment domains of WPAI:SHP in biologic-naïve or TNFi-experienced patients (inadequate responders to TNFi or TNFi-intolerant patients) with PsA

\begin{tabular}{|c|c|c|c|c|c|c|c|c|}
\hline & \multicolumn{4}{|c|}{ Biologic-naïve patients } & \multicolumn{4}{|c|}{ TNFi-experienced patients } \\
\hline & $\begin{array}{l}\text { Baseline } \\
\text { S.D. }\end{array}$ & Reliability $^{a}$ & $0.5 \times$ S.D. & S.E.M. & $\begin{array}{l}\text { Baseline } \\
\text { S.D. }\end{array}$ & Reliability $^{\mathrm{a}}$ & $0.5 \times$ S.D. & S.E.M. \\
\hline \multicolumn{9}{|l|}{ WPAI:SHP domain } \\
\hline Presenteeism & 23.71 & 0.7 & 11.85 & 12.99 & 26.59 & 0.7 & 13.29 & 14.56 \\
\hline $\begin{array}{l}\text { Work productivity } \\
\text { loss }\end{array}$ & 24.88 & 0.7 & 12.44 & 13.63 & 27.69 & 0.7 & 13.85 & 15.17 \\
\hline $\begin{array}{l}\text { Activity } \\
\text { impairment }\end{array}$ & 25.01 & 0.7 & 12.51 & 13.70 & 25.77 & 0.7 & 12.89 & 14.12 \\
\hline
\end{tabular}

TNFi tumor necrosis factor inhibitor, WPAI:SHP Work Productivity and Activity Impairment: Specific Health Problem Questionnaire, S.D. standard deviation, S.E.M. standard error of measurement

${ }^{a}$ As reported by Crawford and colleagues [20]

that patients with PsA have major joint symptoms that may or may not be accompanied by skin symptoms, while patients with psoriasis have major skin symptoms that may or may not be accompanied by joint symptoms. Similarly, the alignment in the estimates for activity impairment reflects the common aspects of the diseases, such as physical and psychosocial burdens or the general impact on health-related quality of life [26].

In this study, we reported estimates for the presenteeism, work productivity, and activity impairment domains of WPAI:SHP in patients with PsA using results from two clinical trials, but we acknowledge that our study has both limitations and strengths. The patient populations of the two trials were selective and consisted predominantly of patients with the polyarthritis phenotype, but this limitation is countered by the numerous strengths associated with these clinical trials, such as well-characterized patient populations, robust data collection, and the inclusion of two distinct populations of biologic-naïve and TNFi-experienced patients. In addition, this study does not report thresholds for deterioration, which may have a different magnitude of change than reported for the improvement in MCIDs. Importantly, the reported MCIDs must be applied to patient-level data, and cannot be compared to population-level data.

\section{CONCLUSION}

In conclusion, using clinical trial data, we estimated MCIDs for the presenteeism, work productivity loss, and activity impairment domains of WPAI:SHP in biologic-naïve and TNFi-experienced patients with PsA. The MCID estimates for biologic-naïve and TNFi-experienced patients were consistent for the two patient populations. The MCID for presenteeism was $20 \%$, the MCID for work productivity loss improvement was $15 \%$, and the MCID for activity impairment improvement was $20 \%$. When applied to patient-level data, these estimates should allow clinicians to more effectively evaluate the effect of treatments on work productivity in biologic-naïve or TNFi-experienced patients with PsA.

\section{ACKNOWLEDGEMENTS}

Funding. This study and article processing charges were funded by Eli Lilly and Company. All authors had full access to all of the data in this study and take complete responsibility for the integrity of the data and accuracy of the data analysis. 
Medical Writing Assistance. Medical writing support in the preparation of this article was provided by Brian S. Comer, Ph.D. of Eli Lilly and Company. Support for this assistance was funded by Eli Lilly and Company.

Authorship. All named authors meet the International Committee of Medical Journal Editors (ICMJE) criteria for authorship for this article, take responsibility for the integrity of the work as a whole, and have given their approval for this version to be published.

Disclosures. William TIllett has received grants/speaker fees and/or honoraria from AbbVie, Celgene, Eli Lilly and Company, Janssen, Novartis, Pfizer, and UCB. Chen-Yen Lin is an employee and shareholder of Eli Lilly and Company. Aubrey Trevelin Sprabery is an employee and shareholder of Eli Lilly and Company. Julie Birt is an employee and shareholder of Eli Lilly and Company. Art Zbrozek is retired and a former employee of Eli Lilly and Company.

Compliance with Ethics Guidelines. Both SPIRIT-P1 and SPIRIT-P2 were conducted in accordance with the ethical principles of the Declaration of Helsinki and in compliance with local laws and regulations. All participants provided informed consent. SPIRIT-P1 and SPIRITP2 protocols and consent forms were approved by each site's institutional review board or ethics committee, including the Western Institutional Review Board for SPIRIT-P1 and the Bellberry Human Research Ethics Committee for SPIRIT-P2. The individual SPIRIT-P1 and SPIRIT-P2 sites are listed in the primary manuscript supplements $[13,14]$. Both studies were registered on ClinicalTrials.gov (SPIRIT-P1: NCT01695239, SPIRIT-P2: NCT02349295).

Data Availability. Eli Lilly provides access to all data on individual participants collected during the trial after anonymization, with the exception of pharmacokinetic or genetic data. Data are available to request 6 months after the indication studied has been approved in the US and EU and after primary publication acceptance, whichever is later. No expiration date of data requests is currently set once they are made available. Access is provided after a proposal has been approved by an independent review committee identified for this purpose and after the receipt of a signed data-sharing agreement. Data and documents, including the study protocol, statistical analysis plan, clinical study report, and blank or annotated case report forms, will be provided in a secure data-sharing environment for up to 2 years per proposal. For details on submitting a request, see the instructions provided at www.clinicalstudydatarequest.com.

Open Access. This article is distributed under the terms of the Creative Commons Attribution-NonCommercial 4.0 International License (http://creativecommons.org/licenses/ by-nc/4.0/), which permits any noncommercial use, distribution, and reproduction in any medium, provided you give appropriate credit to the original author(s) and the source, provide a link to the Creative Commons license, and indicate if changes were made.

\section{REFERENCES}

1. de Vlam K, Gottlieb AB, Mease PJ. Current concepts in psoriatic arthritis: pathogenesis and management. Acta Derm Venereol. 2014;94(6):627-34.

2. Ritchlin CT, Colbert RA, Gladman DD. Psoriatic arthritis. N Engl J Med. 2017;376(10):957-70.

3. Tillett W, de Vries C, McHugh NJ. Work disability in psoriatic arthritis: a systematic review. Rheumatology (Oxford). 2012;51(2):275-83.

4. Bojke L, Spackman E, Hinde S, Helliwell P. Capturing all of the costs in NICE appraisals: the impact of inflammatory rheumatic diseases on productivity. Rheumatology (Oxford). 2012;51(2):210-5.

5. Kawalec P, Malinowski KP. The indirect costs of psoriatic arthritis: systematic review and metaanalysis. Expert Rev Pharmacoecon Outcomes Res. 2015;15(1):125-32.

6. Lee S, Mendelsohn A, Sarnes E. The burden of psoriatic arthritis: a literature review from a global health systems perspective. Pharm Ther. 2010;35(12):680-9. 
7. Reilly MC, Zbrozek AS, Dukes EM. The validity and reproducibility of a work productivity and activity impairment instrument. Pharmacoeconomics. 1993;4(5):353-65.

8. Reilly Associates. WPAI:SHP V2.0 (updated 8/18/ 2010). Available from: http://www.reillyassociates. net/WPAI_SHP.html. Accessed 15 Sep 2017.

9. Gottlieb AB, Mease PJ, Cuchacovich RS, Shuler CL, Lin C-Y, Burge RT, et al. Ixekizumab improves physical function, quality of life, and work productivity in biologic disease-modifying antirheumatic drug-naive patients with active psoriatic arthritis [abstract]. Arthritis Rheumatol. 2015;67(Suppl 10):A2145.

10. Kavanaugh A, Vender R, Birt J, Adams D, Benichou $\mathrm{O}$, Lin C-Y, et al. SAT0446 Ixekizumab improves patient-reported outcomes in patients with active psoriatic arthritis and previous inadequate response to tumour necrosis factor-inhibitors. Ann Rheum Dis. 2017;76(Suppl 2):941.

11. Tillett W, Shaddick G, Askari A, Cooper A, Creamer $P$, Clunie $G$, et al. Factors influencing work disability in psoriatic arthritis: first results from a large UK multicentre study. Rheumatology (Oxford). 2015;54(1):157-62.

12. Jayadevappa R, Cook R, Chhatre S. Minimal important difference to infer changes in health-related quality of life-a systematic review. J Clin Epidemiol. 2017;89:188-98.

13. Mease PJ, van der Heijde D, Ritchlin CT, Okada M, Cuchacovich RS, Shuler CL, et al. Ixekizumab, an interleukin-17A specific monoclonal antibody, for the treatment of biologic-naive patients with active psoriatic arthritis: results from the 24-week randomised, double-blind, placebo-controlled and active (adalimumab)-controlled period of the phase III trial SPIRIT-P1. Ann Rheum Dis. 2017;76(1):79-87.

14. Nash P, Kirkham B, Okada M, Rahman P, Combe B, Burmester GR, et al. Ixekizumab for the treatment of patients with active psoriatic arthritis and an inadequate response to tumour necrosis factor inhibitors: results from the 24-week randomised, double-blind, placebo-controlled period of the SPIRIT-P2 phase 3 trial. Lancet (London, England). 2017;389(10086):2317-27.

15. FDA. Guidance for industry patient-reported outcome measures: use in medical product development to support labeling claims. Washington, DC: US Department of Health and Human Sciences; 2009.

16. Copay AG, Subach BR, Glassman SD, Polly DW Jr, Schuler TC. Understanding the minimum clinically important difference: a review of concepts and methods. Spine J. 2007;7(5):541-6.

17. Coates LC, Fransen J, Helliwell PS. Defining minimal disease activity in psoriatic arthritis: a proposed objective target for treatment. Ann Rheum Dis. 2010;69(1):48-53.

18. Coates LC, Husni ME, Shuler CL, Carlier H, Lin C-Y, Mou J, et al. THU0440 Ixekizumab provides sustained improvement up to 52 weeks of disease activity as assessed by composite measure scores in biologic disease-modifying antirheumatic drug (bDMARD)-naive patients with active psoriatic arthritis. Ann Rheum Dis. 2016;75(Suppl 2):349-50.

19. Hays RD, Farivar SS, Liu H. Approaches and recommendations for estimating minimally important differences for health-related quality of life measures. COPD. 2005;2(1):63-7.

20. Crawford B, Okuda M, Leahy M. The RQLQ and WPAI-AS questionnaires are valid, reliable measures of QOL in Japanese patients with seasonal allergic rhinitis. Presented at: ISPOR First Asia-Pacific Conference; 2003 Sept 1-3; Kobe, Japan.

21. Norman GR, Sloan JA, Wyrwich KW. Interpretation of changes in health-related quality of life: the remarkable universality of half a standard deviation. Med Care. 2003;41(5):582-92.

22. Crosby RD, Kolotkin RL, Williams GR. Defining clinically meaningful change in health-related quality of life. J Clin Epidemiol. 2003;56(5):395-407.

23. Armstrong AW, Lynde CW, McBride SR, Stahle M, Edson-Heredia E, Zhu B, et al. Effect of ixekizumab treatment on work productivity for patients with moderate-to-severe plaque psoriasis: analysis of results from 3 randomized phase 3 clinical trials. JAMA Dermatol. 2016;152(6):661-9.

24. Bureau of Labor Statistics. May 2016 national occupational employment and wage estimatesUnited States. Washington, DC: US Department of Labor; 2016. Available from: https://www.bls.gov/ oes/current/oes_nat.htm\#00-0000. Accessed 15 Sep 2017.

25. Wu J, Lin C-Y, Sun L, Goldblum O, Zbrozek A, Russel B. Minimal clinically important difference for work productivity and activity impairment questionnaire in psoriasis patients. J Manag Care Spec Pharm. 2017;23(10-a):S70.

26. Mease PJ, Menter MA. Quality-of-life issues in psoriasis and psoriatic arthritis: outcome measures and therapies from a dermatological perspective. J Am Acad Dermatol. 2006;54(4):685-704. 